\title{
Synthesis of thiophene-benzodithiophene wide bandgap polymer and GIWAXS evaluation of thermal annealing with potential for application in ternary polymer solar cells
}

JOSE JONATHAN RUBIO ARIAS ${ }^{l}$ jonathanrubio@ima.ufrj.br

ISABELA CUSTÓDIO MOTA ${ }^{1}$ isabelacmota@ima.ufrj.br

MARIA DE FÁTIMA VIEIRA MARQUES ${ }^{l}$ fmarques@ima.ufrj.br

1. Universidade Federal do Rio de Janeiro, Instituto de Macromoléculas Eloisa Mano, IMA-UFRJ. Cidade Universitária. Av. Horácio Macedo, 2.030. Centro de Tecnologia. Bloco J. Rio de JaneiroRJ. Brazil.

\begin{abstract}
Conjugated polymers are a special type of material responsible for light absorption in polymer solar cells. The absorption of these materials plays an important role in photon harvesting and energy conversion. Ternary organic solar cells are a type of OPV that combines two-electron donors with complementary absorption in a single layer. The present work deals with the synthesis and characterization of a high bandgap polymer prepared through the alternation of two high electron density aromatic units (thiophenebenzodithiophene). This polymer presented high bandgap $(2.1 \mathrm{eV})$ with potential for complementary absorption in a ternary device, as well as high $\mathrm{V}_{\text {oc }}$ when applied in a single junction inverted OPV device in heterojunction with $\mathrm{PC}_{70} \mathrm{BM}(0.95 \mathrm{~V})$; which agrees with the relatively large energy difference between its $\mathrm{HOMO}$ and the LUMO of $\mathrm{PC}_{70} \mathrm{BM}$ determined through CV and UV-vis. A dynamic GIWAXS study was performed and demonstrated that for this polymer, increasing annealing temperature in $80{ }^{\circ} \mathrm{C}$ can increase its crystalline organization by up to $50 \%$.
\end{abstract}

Keywords: Benzodithiophene Conjugated polymer; GIWAXS; Polymer solar cell

\section{Introduction}

Solar energy is one of the cleanest alternatives in the current world energy matrix, and it is expected to have a constant growth during the next years, due to its high potential for energy conversion without need of fuels and zero $\mathrm{CO}_{2}$ emissions during use [1,2]. Organic photovoltaic devices in which conjugated polymers compose the active layer are one of the ways wherein solar energy is expected to increase its impact in the energy market due to its high versatility, lightweight, and relative ease of processing [3]. An organic solar cell is composed of several layers with different properties, highlighting the active layer, where light excites the electrons of a photoactive conjugated polymer (electron donor), which transfers this excited electrons to an electron acceptor (either conjugated polymer or fullerene derivative PCBM); electron donor and 
acceptor form the as-called bulk heterojunction (BHJ), that composes the main focus of attention in polymer research, since it is aimed to reach polymer structures with broader absorption spectra while its structure renders proper BHJ morphology that allows maximum charge transfer and minimum energy losses $[3,4]$. The BHJ solar cells could be greatly improved when absorption spectrum of polymers are broad, however, tailoring electron donors to a very broad absorption spectrum can be tricky, involving very complicated structures that make the material to be too expensive, such as copolymers of polythiophenes with bithiophene grafted polythiophenes [5] or a A-D copolymers of alkylthiophene-substituted benzodithiophene and thiazolo[5,4-d]thiazole (TTZ) units [6], among others.

Ternary solar cells are an alternative for diminishing the mismatch between the solar spectrum and the light absorption of the active layer by adding complementary absorptions of a wide and a narrow bandgap polymer [7-9]. On the other hand, ternary solar cells can also comprehend the inclusion of two electron acceptors with the function of supplementing absorption, reducing energy loss $[10,11]$ and inducing an improved morphology of the BHJ [12] .

Narrow bandgap polymers are materials that have gained much attention due to their absorption edge close to the infrared region and consequently improved photon harvesting [13]; nevertheless, this polymers could present low absorption in the medium region of the visible spectrum (380-600 nm) [14]. Thus, the present work explores the synthesis and characterization of a relatively simple wide bandgap polymer prepared by the condensation of two aromatic units of high electron density, benzodithiophene (BDT) and thiophene (Th). A similar structure has been already synthesized by Hou and co-workers in 2008 [15], and bandgap was reported to be wide $(>2 \mathrm{eV})$. One of the advantages of a BDT-Th is that its relative simplicity compared to the already mentioned structures [5,6], can be a path to follow in the search for broader absorption in BHJ solar cells. While Hou's polymer structure had a dodecyl linear chain attached to the BDT unit, our proposed structure has a 2-butyloctyl chain, which will allow to verify if the ramification has an actual impact on absorption and other properties of the polymer. The polymer was synthesized via the Stille polymerization mechanism through the coupling of high electron density units, which, as it has been previously established, determines the absorption behavior of conjugated polymers $[13,16]$.

\section{Experimental}

\subsection{Materials}

Synthesis and characterization of monomer 2,6-bis(trimethylstannyl)-4,8-bis(butylhexyloxy)benzo[1,2b:4,5-b'] dithiophene (M1) is fully described somewhere else [14].

Commercial ITO plates and $\mathrm{ZnO}$ dispersion were provided by Ossila. Monomer 2,5-dibromothiophene (M2), and tetrakistriphenylphosphine palladium (0), $\mathrm{Pd}\left(\mathrm{PPh}_{3}\right)_{4}$, were purchased from TCI chemicals Japan. 
Deuterated chloroform was acquired from TCI Chemicals, Japan. All reagents were used as received. Toluene was dried by pre-treatment with molecular sieves and distillation over Na-benzophenone.

\subsection{Polymer synthesis}

The polymer was synthesized under an inert atmosphere using Schlenk technique, by mixing $0.360 \mathrm{~g}(0.5$ $\mathrm{mmol})$ of $\mathrm{M} 1,0.121 \mathrm{~g}(0.5 \mathrm{mmol})$ of $\mathrm{M} 2$ and $5.8 \mathrm{mg}$ of $\mathrm{Pd}\left(\mathrm{PPh}_{3}\right)_{4}$ in dry toluene, degassing by fluxing nitrogen for $30 \mathrm{~min}$ and heating under magnetic stirring for $48 \mathrm{~h}$ at reflux temperature. After the reaction time, the product was precipitated into cold, well-stirred methanol, washed and submitted to Soxhlet extractions with methanol, acetone, hexane, and chloroform, which produced a red-pink solution that was precipitated in methanol and the solid was filtered and dried overnight under vacuum at $60^{\circ} \mathrm{C}$.

\subsection{Characterization techniques}

\section{Gel Permeation Chromatography}

Gel permeation chromatography (GPC) was run in a Prominence® UFLC GPC equipment from Shimadzu, equipped with two columns as follow: 1) Shim-pack GPC-803C, 300 x $8.0 \mathrm{~mm}$ exclusion limit (MW): $7 \mathrm{x}$ $10^{4}$ and 2) Phenogel ${ }^{\mathrm{TM}} 5 \mu \mathrm{m}$ Linear, $300 \times 7.8 \mathrm{~mm}$, exclusion limit (MW): $1 \times 10^{7}$. Chloroform was used as eluent, while a refraction index detector was equipped for fractions detection.

Thermogravimetric analysis

A Q500 V20.13 Build 39 from TA Instruments was used for TGA analyses at $10{ }^{\circ} \mathrm{C} / \mathrm{min}$ heating rate between 30 and $700{ }^{\circ} \mathrm{C}$ using nitrogen as carrier gas $(60 \mathrm{ml} / \mathrm{min})$.

\section{Fourier Transform Infrared Spectroscopy}

FTIR spectra were recorded using an Excalibur 3100 IR from VARIAN in attenuated total reflectance (ATR) mode using the corresponding ATR accessory from Varian. The analytic procedure was 50 scans, resolution of $2 \mathrm{~cm}^{-1}$ in the range $5000-600 \mathrm{~cm}^{-1}$.

\section{Nuclear magnetic resonance}

A Varian M400 NMR equipment with a $400 \mathrm{MHz}$ (9.4 Tesla) Premium Shielded Magnet was used for solution ${ }^{1}$ HNMR. The analyses were performed using $5 \mathrm{~mm}$ tubes with samples diluted in deuterated chloroform.

\section{$U V$-Vis spectroscopy}

Measurements were performed in a CARY 1000 UV-vis spectrophotometer in the region between 280 and $900 \mathrm{~nm}$ at a rate of $5 \mathrm{~nm} / \mathrm{s}$. Samples consisted of thin films obtained by spin-coating deposition of polymer solutions $(2 \mathrm{mg} / \mathrm{mL}$ ) onto glass plates. Equation 1 was used to obtain the energy bandgap (Eg) from absorption onset, where $\mathrm{h}$ and $\mathrm{c}$ are the Planck constant and light speed in a vacuum, respectively.

$$
E_{g}=c * h / \lambda_{\text {onset }} \quad \text { Equation } 1
$$

Grazing Incidence Wide Angle X-ray scattering 
GIWAXS experiments were performed at the XRD2 beamline, located at the Brazilian Synchrotron Light Laboratory (LNLS/CNPEM), using beam energy of $8 \mathrm{keV}\left(\lambda=0.155 \mathrm{~nm}^{-1}\right)$. The scattered intensity was collected using a Pilatus 300k detector (Dectris, Switzerland) positioned $240 \mathrm{~mm}$ from the sample, collecting data from $s$ values between 2 and 17. Data reduction was made by the use of Fit2D. The measurements were performed at different temperatures between 140 and $240{ }^{\circ} \mathrm{C}$ for glass plates spun with polymer solution in dichlorobenzene $(15 \mathrm{mg} / \mathrm{mL})$. Figure S.I.1 shows a collage of pictures illustrating the experimental setup used for the GIWAXS analyses, where the home-made furnace, the detector and the localization of the sample in the system can be identified. The sample was scanned at ten different temperatures ranging from 140 to $240{ }^{\circ} \mathrm{C}$ (six scans at each temperature). Atmospheric conditions were used for the analyses, and the coated plates were not submitted to any treatment after deposition, looking for observing morphology changes caused solely by the variation of the temperature inside the home-made furnace.

\section{Cyclic voltammetry}

The analysis of cyclic voltammetry was performed according to the procedure reported somewhere else [14], through which it is possible to obtain HOMO and LUMO levels of synthesized polymers through the use of Equation 2 and 3, where Ered and Eox are the onsets of reduction and oxidation processes during the scan.

$$
\begin{array}{ll}
E_{\text {Номо }}=-e[\text { Ered }+4.4] & \text { Equation 2 } \\
E_{\text {LUMO }}=-e[\text { Eox }+4.4] & \text { Equation 3 }
\end{array}
$$

\subsection{Device Fabrication and Evaluation}

\section{Active layer solution}

Active layer was prepared by weighting equal masses of polymer and $\mathrm{PC}_{70} \mathrm{BM}$ in a microbalance in open atmosphere, poured inside a small vial and taken to glovebox, where dry dichlorobenzene was added to produce a $30 \mathrm{mg} / \mathrm{mL}$ solution of electron donor and acceptor (1:1), which was stirred overnight and filtered through a $13 \mu \mathrm{m}$ PVDF hydrophobic filter and stored until deposition.

\section{Layers deposition}

Photovoltaic devices were produced according to the experimental procedure shown in Scheme 2, starting from an ITO plate, which was previously cleaned as described in Supporting Information (S.I) document. In a glovebox environment, filled with nitrogen, a solution of $\mathrm{ZnO}$ precursor was spin-coated onto the ITO plate. It was baked at $70{ }^{\circ} \mathrm{C}$ in regular atmosphere, after which the plate was returned to the glovebox, and the active layer solution was spin-coated at $900 \mathrm{rpm}$ for $3 \mathrm{~min}$, followed by thermal annealing at $140{ }^{\circ} \mathrm{C}$ for 
20 min without any additives. A corner of the plate was cleaned to expose the ITO surface. Then, $\mathrm{MoO}_{3}$ deposition was carried out using a round-shaped $1 \mathrm{~mm}^{2}$ holes mask, which permitted to obtain several 1 $\mathrm{mm}^{2}$ devices after the silver thermal deposition. Cathode (the ITO exposed face) and anode (the silver surface), were connected to the apparatus for efficiency measurement. Devices were tested using a solar lamp equipped with an AM1.5G filter. PCE estimation was done as reported somewhere else [14].

\section{Results and discussion}

The synthesis of PBOBDTTh polymer was performed as previously described. The proposed mechanism (Figure 1) involves the formation of intermediary palladium species containing both the benzodithiophene (BOBDT) and Thiophene (Th) units. It was previously reported [17-19] that this catalyst is active for C-C coupling solely above $80^{\circ} \mathrm{C}$, which was achieved for the present reaction, carried out at reflux temperature. The slow transition of reaction mixture color from transparent to red-purple, passing through yellow and orange, as well as the viscosity gain, was accounted as clear evidence of chain growth. After the purification process, precipitation and drying, $0.190 \mathrm{~g}$ (60\% yield) of a bright green-reddish soft solid was separated (Its physical aspect is shown in Figure 2).

\subsection{Functional and thermal characterization}

The proton nuclear magnetic resonance $\left({ }^{1} \mathrm{HNMR}\right)$ in Figure 3 displays the spectra of the precursor monomer (BOBDT) and the resulting PBOBDTTh polymer. The primary evidence for reaction provided is the absence of the peaks assigned as (a) in the region between 0.8 and $2 \mathrm{ppm}$ of the polymer spectrum, which evidences the consumption of all the active sites containing the trimethyl tin moiety. It is observed that the $\mathrm{CDCl}_{3}$ peak is located at the same position in both spectra; however, a clear shift of the benzodithiophene protons for lower ppm values can be noticed, which is ascribed to the modification of chemical environment caused by backbone growth. Another significant effect is the overlapping of aromatic protons in regions around $7.20 \mathrm{ppm}$, which caused the overlapping with the $\mathrm{CDCl}_{3}$ peak itself. It is worth to highlight how peaks are extended and less resolved in the polymer, which is another evidence of polymerization reaction as broadly reported in the literature [20-25]. Gel permeation chromatography (GPC) showed that the obtained solid has number average molar mass $\left(M_{n}\right)$ of $34.7 \mathrm{~kg} / \mathrm{mol}$ and dispersity (Đ) of 2.84. The high dispersity value agrees with other literature reports for the Stille mechanism [26,27], and the consequences of this dispersity will be further discussed. 
The thermal stability of the formed polymer was analyzed through TGA (Figure 4). The polymer is stable up to $288^{\circ} \mathrm{C}$, where its mass starts to decay $\left(\mathrm{T}_{\text {onset }}\right)$. Its maximum degradation temperature $\left(\mathrm{T}_{\max }\right)$ is $360{ }^{\circ} \mathrm{C}$, while its $\mathrm{T}_{\text {endset }}$ is $417{ }^{\circ} \mathrm{C}$. Despite possessing lower thermal degradation temperature than a reference polymer such as P3HT [28,29], the obtained PBOBDTTh is sufficiently stable to resist the R2R processing used for OPV devices fabrication [30,31].

\subsection{Characterization of energy levels}

Figure 5 presents a comparison between the UV-vis absorption of a PBOBDTTh thin film and the solar spectrum AM1.5, where the difference between solid-state polymer absorption and solar irradiance spectrum can be found. Absorption edge is found at $592.9 \mathrm{~nm}$, which, by applying Equation 1, permits to determine an optical bandgap of $2.1 \mathrm{eV}$, slightly higher than the one found by Hou [15] of $2.06 \mathrm{eV}$; the very narrow difference permits to infer that the ramification present in the synthesized polymer does not really affect the bandgap of the polymer. It was also observed that $58.2 \%$ of incident visible sunlight fits the absorption by the polymer; despite being a low value compared to other polymers such as P3HT, the absorption behavior of PBOBDTTh is proper for devices in which a mixture of polymers is used (ternary devices), due to its narrow absorption that permits the absorption of a low bandgap polymer, having complementary absorption rather than competitive absorption [32].

Two redox stages were detected in the Cyclic voltammetry (CV) presented in Figure 6. The oxidation onset was estimated as $1.2 \mathrm{~V}$ through graphic interpolation, while the reduction onset was estimated in $-0.67 \mathrm{eV}$ using the same graphic approach. The obtained values of redox onsets can be associated with electron affinity and oxidation potential, which are considered to be equivalent to LUMO and HOMO, respectively [33]. To transform those values in voltage into the energy of LUMO and HOMO levels, Equations 2 and 3 were employed, rendering LUMO level of $-3.73 \mathrm{eV}$ and HOMO level of $-5.6 \mathrm{eV}$, substantially diverging from those reported by Hou [15]. As reported in the literature [14,34] the conjugation of cyclic voltammetry and UV-vis spectrometry is a reliable way to determine LUMO level because reduction onset determination in $\mathrm{CV}$ is considered to be less precise, thus, after simple mathematical treatment, the LUMO energy of PBOBDTTh can be calculated by the addition of HOMO level determined through CV and the optical bandgap determined through UV-vis spectrum; thus, the energy levels of the PBOBDTTh were assessed 
and are shown as inset image in Figure 7, along with the energy levels of the subsequent layers [35,36] needed to build an inverted device with the architecture proposed in the experimental section.

Energy levels alignment shows that inverted devices fabricated with PBOBDTTh are feasible to generate photocurrent. Since there is a great difference between LUMO levels of electron donor and an electron acceptor $(0.4 \mathrm{eV})$, it is more likely the exciton dissociation against exciton recombination, which could play an essential role on device performance and quantum efficiency [37]. The energy levels of all the layers show the cascade effect throughout the device from the metallic electrode to the transparent one, which means that, in theory, an inverted device with the architecture ITO/ZnO/PC70BM:PBOBDTTh/ $\mathrm{MoO}_{3} / \mathrm{Ag}$ must work properly.

\subsection{Device Fabrication and evaluation}

After verifying the compatibility of energy levels in an inverted device (ITO/ZnO/PC $70 \mathrm{BM}: \mathrm{PBOBDTTh} / \mathrm{MoO}_{3} / \mathrm{Ag}$ ), devices were fabricated as described in materials and methods section, obtaining several devices of $1 \mathrm{~mm}$ area per ITO plate. One of the major issues found was the deposition of the $\mathrm{ZnO}$ layer, which sometimes is not efficient due to the viscosity of the solution and the high rotation speeds needed to coat the plate properly, which caused that a high fraction of devices did not work accurately. With this in mind, the best $1 \mathrm{~mm}$ device is reported, and its plot of $\log (\mathrm{I}) v s$. V is showed in Figure 7. This graphic permits to identify the point in which the current is minimum, that is to say, the point in which the circuit is open. The plot permitted to determine that a non-illuminated device has near zero open-circuit voltage $\left(\mathrm{V}_{\mathrm{oc}}\right)$, while the illuminated one has a $\mathrm{V}_{\mathrm{oc}}$ of $0.95 \mathrm{~V}$, significantly higher than the ones reported in the literature for P3HT and low-bandgap polymer-based devices [38,39]. This larger $\mathrm{V}_{\text {oc }}$ could be explained both in the large energy difference between LUMO of acceptor and HOMO of the donor, which is directly correlated to energy gain since that is the actual energy obtained by an excited photoelectron when transferred to an electron acceptor. This value is higher than the one found for an analogous BDT-Th structure synthesized by Huo [15] of 0.75 , and this difference could be attributed to the side chain, that for being so long in the case of Hou's polymer, could induce torsions in the molecule, that end up diminishing its Voc. However, the trend in both cases is to obtain high Voc values from coupling of BDT and Th, since the analogous structures [15] that consisted in other aromatic units coupled to BDT, presented lower Voc values and lower bandgaps.

Photovoltaic parameters were determined from the JV curve of the inverted device fabricated with PBOBDTTh showed in Figure 8. PBOBDTTh presented a relatively low fill factor of $41.5 \%$, lower than the typical values above $50 \%$ found in the literature for P3HT and low-bandgap polymers. This low value 
maybe also explained in the lack of layer thickness optimization, which caused a low current density (-1.96 $\mathrm{mA} / \mathrm{cm}^{2}$ ) and low shunt resistance (high decrease rate of $\mathrm{J}$ along voltage increase).

It would be necessary to optimize the thickness of this device in order to make it achieve higher currents and FF values, which will render higher PCE values. Maximum power was achieved at $0.65 \mathrm{~V}$, which is significantly higher than the values found in the literature, and it also occurs to $V_{\text {oc }}$ value, which is high $(0.95 \mathrm{~V})$ for the analyzed polymer. At the same time, it is lower for reference polymers in inverted devices, such as P3HT or PTB7 [40,41]. Taking into consideration that the UV-vis spectrum showed a low fraction of absorbed sunlight for PBOBDTTh (less than 59\%), it can be inferred that this polymer would present, even without optimization, a high value of Internal Quantum Efficiency, which should be verified in future works. A PCE of $0.75 \%$ was obtained for PBOBDTTh; this value is low when compared to other polymers such as P3HT [42] and lower than the obtained by Hou [15] (1.6\%). All the main features of the synthesized polymer are shown in Table 1, from thermal stability to photovoltaic parameters.

Despite the relatively low PCE obtained for the studied polymer as aforementioned, several improvements such as thickness optimization, which is expected to produce an increase of $\mathbf{J}_{\mathrm{sc}}$ and FF; electron acceptor type and concentration, thermal annealing, solvent annealing, additives and the inclusion of a second complementary electron donor can be performed in order to obtain higher PCE values for inverted devices containing PBOBDTTh. The potential for this polymer in ternary devices is reflected in the high energy gain demonstrated by its high Voc and complementary absorption to low band-gap polymers.

\subsection{Thermal annealing and crystallization via GIWAXS}

Taking into consideration the data presented in table 1 and the potential of PBOBDTTh for optimization, this work presents a study on thermal annealing to improve solid-state organization and, thus, possibly improve the PCE values. For this purpose, Grazing Incidence Wide Angle X-Ray scattering was performed in thin films of pure polymer deposited on glass substrates. The details of the experiment are presented in the experimental section.

Figure 9 presents the time evolution of the peak observed at low angles, which, as reported by previous works, correspond to the interplanar distance ruled by the alkyl chain length $[14,43,44]$, as it is depicted in the same figure as inset image. It is important to highlight that this peak at low angles was chosen because it shows the most significant modification along with thermal treatment, which permitted to conclude about the crystalline organization in the thin film solid form. As temperature increases, it was observed an increase in the intensity and area of the diffraction peak, which seems to be less significant above $200{ }^{\circ} \mathrm{C}$. Inset 
images of scattering patterns also confirm the modification of the scattering pattern at low angles along with the heating treatment.

In order to better study and quantify the variation of the peaks presented in Figure 9, gaussian deconvolutions were applied over every experiment performed at each temperature, which rendered the multiple plots in Figure 10, where there are depicted the evolution of peak area (A), peak maximum (Xc) and full width at half maximum (FWHM) as a function of temperature, including the error bars that reflect the high repeatability of measurements. There was a slight overall decrease on the peak maximum (Xc) along with the whole experiment, where its maximum value was 3.46 while its minimum was 3.39 (2.02\% of its initial value); such a small variation permits to infer that above $140{ }^{\circ} \mathrm{C}$, the increase of interplanar distance is almost neglectable and remains virtually constant.

The behavior of the peak area with the increase of temperature reveals a significant increase as it can be observed in Figure 10 (red curve). It was observed a 51.6\% total difference in peak area induced by the thermal treatment. Up to $220^{\circ} \mathrm{C}$, the change of peak area becomes low enough to be ignored; thus, one can say that the best crystalline organization for this polymer is reached after $10 \mathrm{~min}$ of thermal annealing at $200{ }^{\circ} \mathrm{C}$. The crystallite size did not seem to vary, which could be explained by the very nature of the backbone, that imposes a limit to the chain folding, restraining, thus the crystallite to grow.

The increase of area between 140 and $240{ }^{\circ} \mathrm{C}$ responds to an exponential equation. Assuming that the increase of the peak area governs crystalline organization in thin solid state film, it is possible to establish the relative crystallinity (compared to the value at $140{ }^{\circ} \mathrm{C}, \mathrm{RC} 140$ ) parameter, which describes the relative degree of crystallinity in solid-state films of PBOBDTTh compared to the one obtained when a solid thin film is annealed at $140^{\circ} \mathrm{C}$; thus, a variable conversion was performed, transforming the values of the area into RC140, which were used to perform a non-linear fitting using an exponential decay function from Origin software (Expdec1), obtaining thus the Equation 4, which can be assumed to describe the crystalline organization (RC140) in thin-film solid-state as a function of temperature (T) in ${ }^{\circ} \mathrm{C}$, and it allows to verify the crystalline organization at intermediary and lower temperatures not covered by the present study.

$R C 140=-4925 e^{-T / 29.5}+144 \quad$ Equation 4

This realization is a significant achievement, for it can be a pathway for new devices based in PBOBDTTh that is potentially more efficient. If we consider that charge carriers' mobility in an OPV's active layer relies mainly on the solid-state organization of electron donors, it could be expected about a 50\% increase of PCE just by applying thermal annealing at the temperatures suggested by the results presented in the 
experiment. This realization would apply not just for this polymer but for others, which will be attempted in future studies.

\section{Outlook and Conclusions}

Throughout the present study, it was presented the synthesis and characterization of a brand-new wide bandgap benzodithiophene polymer. This structure showed proper thermal behavior for R2R processing techniques and suitable molar mass to achieve thin films that rendered functional binary devices. The polymer devices (using PC70BM as acceptor) showed high open-circuit voltage (0.95 V). Nevertheless, $\mathbf{J}_{\mathrm{sc}}$ was low, and FF was under 50\%, which led to a relatively low PCE of $0.75 \%$. Despite high energy gain reflected in the great difference between HOMO of PBOBDTTh e LUMO of PC70BM, confirmed by the high $\mathrm{V}_{\mathrm{oc}}$, the efficiency is not high, partially due to the low fraction of solar spectrum absorbed by the polymer. One interesting alternative proposed in this work is the utilization of PBOBDTTh as a secondary donor in a ternary device in which its absorption can be complementary to a low-bandgap polymer. Other optimizations for increasing PCE of a single PBOBDTTh device still need to be performed, among which, thermal annealing at high temperatures shows high potential, as disclosed by the dynamic GIWAXS study, which estimates that at least $50 \%$ increase of crystallinity in thin-film solid form can be expected after short thermal annealing at $220^{\circ} \mathrm{C}$.

\section{Acknowledgments}

This research used resources of the Brazilian Synchrotron Light Laboratory (LNLS), an open national facility operated by the Brazilian Center for Research in Energy and Materials (CNPEM), supported by the Brazilian Ministry of Science, Technology, Innovations, and Communications (MCTIC). The XRD2 beamline staff is acknowledged for the assistance during the experiments, especially Dr. Antonio Gasperini, who made possible data processing. We especially acknowledge Prof. Jinsang Kim and dr. Da Seul Yang at the University of Michigan for their support during device fabrication. This study was financially supported in part by the Coordenação de Aperfeiçoamento de Pessoal de Nível Superior - Brazil (CAPES) - Finance Code 001. We would like to thank CNPQ and FAPERJ for their support. We acknowledge the use of FIT2d software, used for GIWAXS data reduction.

\section{References}


[1] J.H. Wee, K.S. Choi, Renew. Sustain. Energy Rev. 14 (2010) 814-820.

[2] F.C. Krebs, M. Jørgensen, K. Norrman, O. Hagemann, J. Alstrup, T.D. Nielsen, J. Fyenbo, K. Larsen, J. Kristensen, Sol. Energy Mater. Sol. Cells 93 (2009) 422-441.

[3] F.C. Krebs, Sol. Energy Mater. Sol. Cells 93 (2009) 394-412.

[4] J.J. Rubio Arias, M. de F. Vieira Marques, React. Funct. Polym. 113 (2017) 58-69.

[5] Y. Li, Y. Zou, Adv. Mater. 20 (2008) 2952-2958.

[6] L. Huo, X. Guo, S. Zhang, Y. Li, J. Hou, Macromolecules 44 (2011) 4035-4037.

[7] P. Cheng, R. Wang, J. Zhu, W. Huang, S.Y. Chang, L. Meng, P. Sun, H.W. Cheng, M. Qin, C. Zhu, X. Zhan, Y. Yang, Adv. Mater. 30 (2018) 1-8.

[8] H. Yin, J.K.W. Ho, S.H. Cheung, R.J. Yan, K.L. Chiu, X. Hao, S.K. So, J. Mater. Chem. A 6 (2018) 8579-8585.

[9] T.M. Grant, T. Gorisse, O. Dautel, G. Wantz, B.H. Lessard, J. Mater. Chem. A 5 (2017) 1581-1587.

[10] R. Yu, S. Zhang, H. Yao, B. Guo, S. Li, H. Zhang, M. Zhang, J. Hou, Adv. Mater. 29 (2017) 1-6.

[11] R. Yu, H. Yao, Z. Chen, J. Xin, L. Hong, Y. Xu, Y. Zu, W. Ma, J. Hou, Adv. Mater. 31 (2019) 18.

[12] Y. Xie, F. Yang, Y. Li, M.A. Uddin, P. Bi, B. Fan, Y. Cai, X. Hao, H.Y. Woo, W. Li, F. Liu, Y. Sun, Adv. Mater. 30 (2018) 1-8.

[13] N.D. Eastham, A.S. Dudnik, B. Harutyunyan, T.J. Aldrich, M.J. Leonardi, E.F. Manley, M.R. Butler, T. Harschneck, M.A. Ratner, L.X. Chen, M.J. Bedzyk, F.S. Melkonyan, A. Facchetti, R.P.H. Chang, T.J. Marks, ACS Energy Lett. 2 (2017) 1690-1697.

[14] J.J.R. Arias, L. Crociani, I.T. Soares, I.C. Mota, B.P.S. Santos, R. Valaski, M.D.F.V. Marques, React. Funct. Polym. 144 (2019) 104355.

[15] J. Hou, M.H. Park, S. Zhang, Y. Yao, L.M. Chen, J.H. Li, Yang, Macromolecules 41 (2008) 60126018.

[16] S. Yang, P. Olishevski, M. Kertesz, Synth. Met. 141 (2004) 171-177.

[17] A.L. Casado, P. Espinet, Organometallics 17 (1998) 954-959.

[18] W.J. Zhou, K.H. Wang, J.X. Wang, J. Org. Chem. 74 (2009) 5599-5602.

[19] E. ichi Negishi, Acc. Chem. Res. 15 (1982) 340-348.

[20] C. Sterling, M. Masuzawa, Die Makromol. Chemie 116 (1968) 140-145.

[21] R.A. Komoroski, J. Polym. Sci. Part A-2, Polym. Phys. 21 (1983) 2551-2559.

[22] M. Zhang, Y. Gu, X. Guo, F. Liu, S. Zhang, L. Huo, T.P. Russell, J. Hou, Adv. Mater. 25 (2013) 4944-4949.

[23] F. Zhang, X. Dai, W. Zhu, H. Chung, Y. Diao, Adv. Mater. 29 (2017) 1-7.

[24] J. Zhao, Y. Li, A. Hunt, J. Zhang, H. Yao, Z. Li, J. Zhang, F. Huang, H. Ade, H. Yan, Adv. Mater. 
28 (2016) 1868-1873.

[25] J. Yuan, X. Huang, H. Dong, J. Lu, T. Yang, Y. Li, A. Gallagher, W. Ma, Org. Electron. Physics, Mater. Appl. 14 (2013) 635-643.

[26] W. Yue, Y. Zhao, S. Shao, H. Tian, Z. Xie, Y. Geng, F. Wang, J. Mater. Chem. 19 (2009) 21992206.

[27] S. Li, L. Ye, W. Zhao, H. Yan, B. Yang, D. Liu, W. Li, H. Ade, J. Hou, J. Am. Chem. Soc. 140 (2018) 7159-7167.

[28] M. Böckmann, T. Schemme, D.H. De Jong, C. Denz, A. Heuer, N.L. Doltsinis, Phys. Chem. Chem. Phys. 17 (2015) 28616-28625.

[29] V. Ho, B.W. Boudouris, B.L. McCulloch, C.G. Shuttle, M. Burkhardt, M.L. Chabinyc, R.A. Segalman, J. Am. Chem. Soc. 133 (2011) 9270-9273.

[30] H.C. Cha, Y.C. Huang, F.H. Hsu, C.M. Chuang, D.H. Lu, C.W. Chou, C.Y. Chen, C.S. Tsao, Sol. Energy Mater. Sol. Cells 130 (2014) 191-198.

[31] H.F. Dam, T.R. Andersen, M. V. Madsen, T.K. Mortensen, M.F. Pedersen, U. Nielsen, F.C. Krebs, Sol. Energy Mater. Sol. Cells 140 (2015) 187-192.

[32] Z. Li, J.D.A. Lin, H. Phan, A. Sharenko, C.M. Proctor, P. Zalar, Z. Chen, A. Facchetti, T.Q. Nguyen, Adv. Funct. Mater. 24 (2014) 6989-6998.

[33] T. Johansson, W. Mammo, M. Svensson, M.R. Andersson, O. Inganäs, J. Mater. Chem. 13 (2003) 1316-1323.

[34] M. Heeney, W. Zhang, D.J. Crouch, M.L. Chabinyc, S. Gordeyev, R. Hamilton, S.J. Higgins, I. McCulloch, P.J. Skabara, D. Sparrowe, S. Tierney, Chem. Commun. (2007) 5061-5063.

[35] G. Garcia-Belmonte, A. Munar, E.M. Barea, J. Bisquert, I. Ugarte, R. Pacios, Org. Electron. 9 (2008) 847-851.

[36] N.S.P. Bhuvanesh, J. Gopalakrishnan, 7 (1997) 2297-2306.

[37] O. Inganäs, M. Berggren, M.R. Andersson, G. Gustafsson, T. Hjertberg, O. Wennerström, P. Dyreklev, M. Granström, Synth. Met. 71 (1995) 2121-2124.

[38] K. Zhao, H. Hu, E. Spada, L.K. Jagadamma, B. Yan, M. Abdelsamie, Y. Yang, L. Yu, R. Munir, R. Li, G.O.N. Ndjawa, A. Amassian, J. Mater. Chem. A 4 (2016) 16036-16046.

[39] Y. Lin, Z.G. Zhang, H. Bai, J. Wang, Y. Yao, Y. Li, D. Zhu, X. Zhan, Energy Environ. Sci. 8 (2015) 610-616.

[40] L. Lu, L. Yu, Adv. Mater. 26 (2014) 4413-4430.

[41] R.S. Loewe, P.C. Ewbank, J. Liu, L. Zhai, R.D. McCullough, Macromolecules 34 (2001) 43244333.

[42] M. Hösel, R.R. Søndergaard, D. Angmo, F.C. Krebs, Adv. Eng. Mater. 15 (2013) 995-1001. 
[43] L. Chen, W. Liu, Y. Yan, X. Su, S. Xiao, X. Lu, C. Uher, X. Tang, J. Mater. Chem. C 7 (2019) 2333-2344.

[44] V. Untilova, T. Biskup, L. Biniek, V. Vijayakumar, M. Brinkmann, Macromolecules 53 (2020) 2441-2453.

\section{Figures and tables}

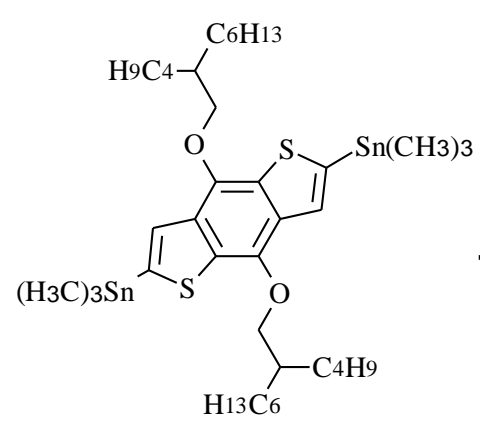

2,6 trimethylthin (4,8-Bis(butyloctyloxy) benzo[1,2-b:4,5-b']dithiophene

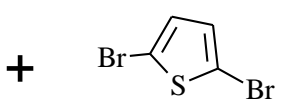

2,5-dibromohexyl thiophene

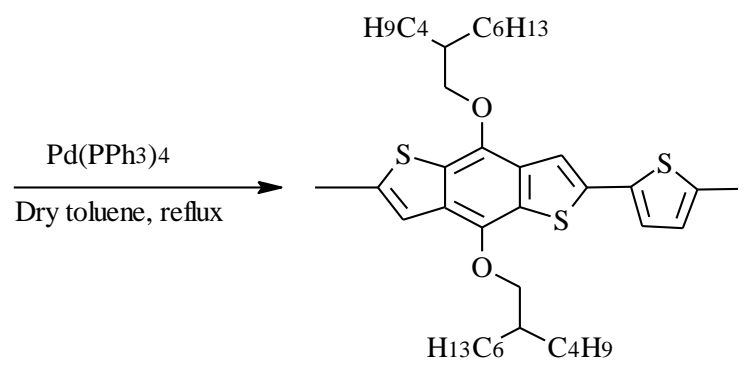

Poly $(2,6(4,8-B i s($ butyloctyloxy) benzo[1,2-b:4,5-b']dithiophene co 2,6-thiophene)

PBOBDTTh

Scheme 1. Reaction scheme and experimental conditions of the polymer proposed in the present work

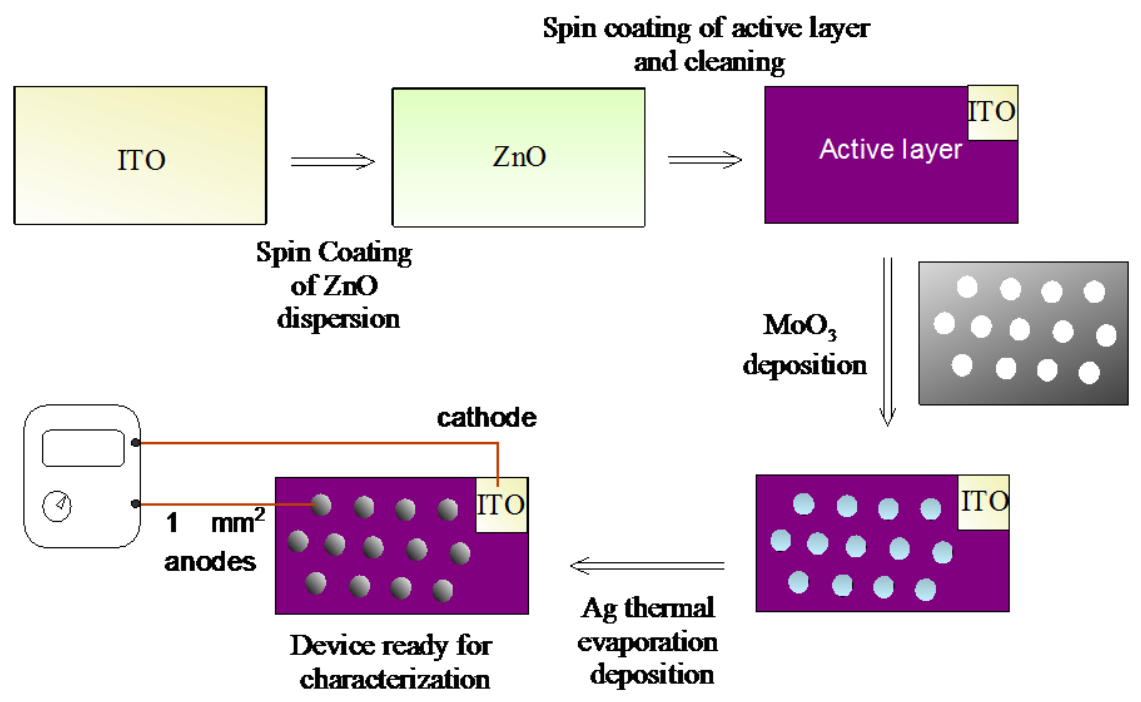

Scheme 2. Device fabrication process used in the present work, which involved the use of round-shaped $1 \mathrm{~mm}^{2}$ holes masks to obtain several $1 \mathrm{~mm}^{2}$ devices inside one single deposition. 


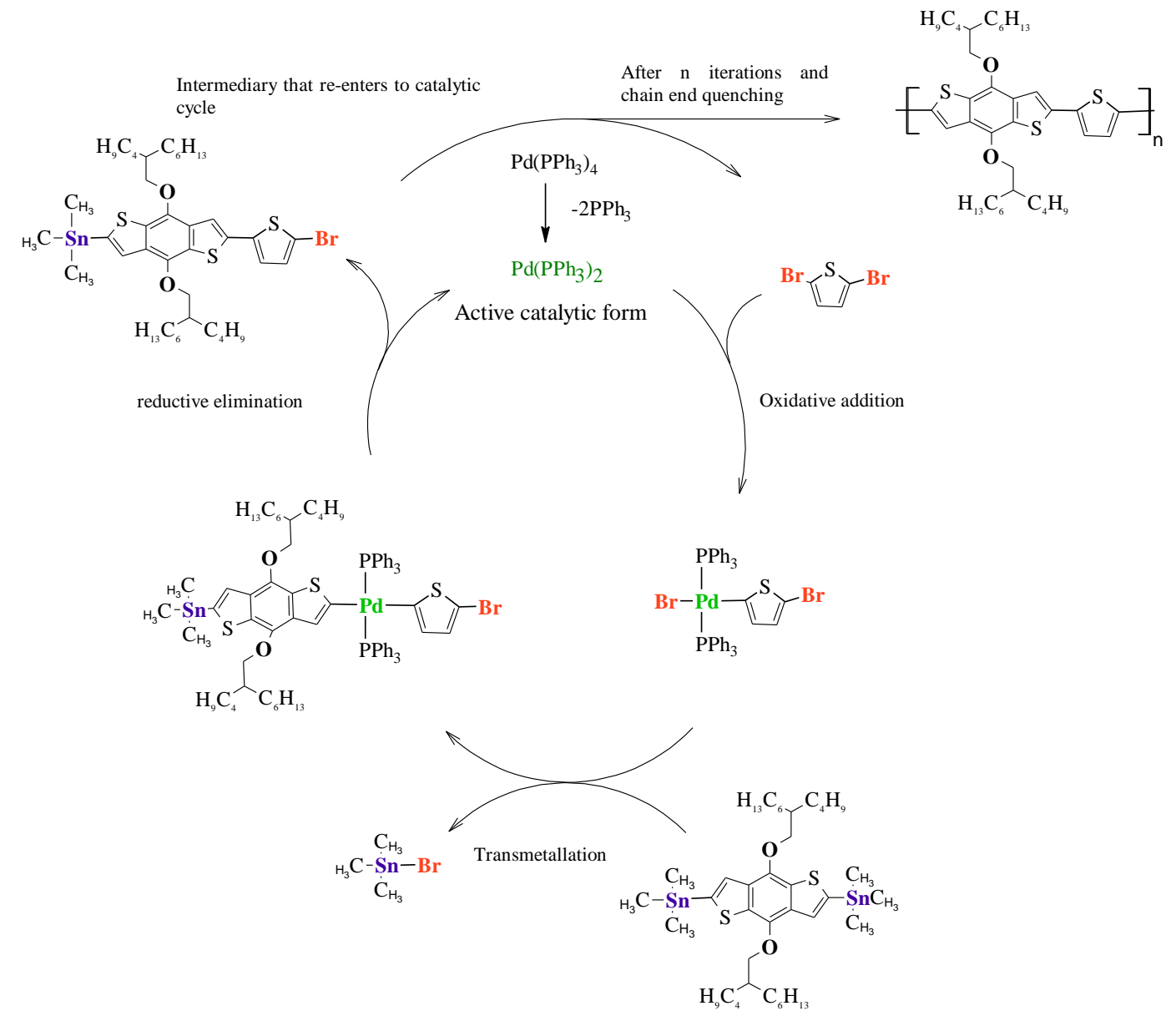

Figure 1. Proposed mechanism for the Palladium mediated polymerization of BOBDT and Th units to produce PBOBDTTh, based on the mechanism for Stille coupling reported somewhere else [17].

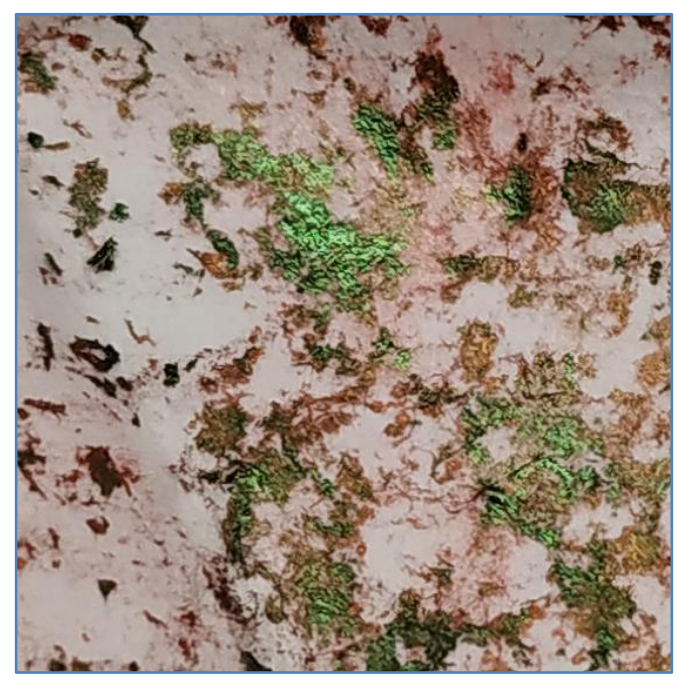

Figure 2. Physical aspect of the obtained green solid after filtration of purified extract 


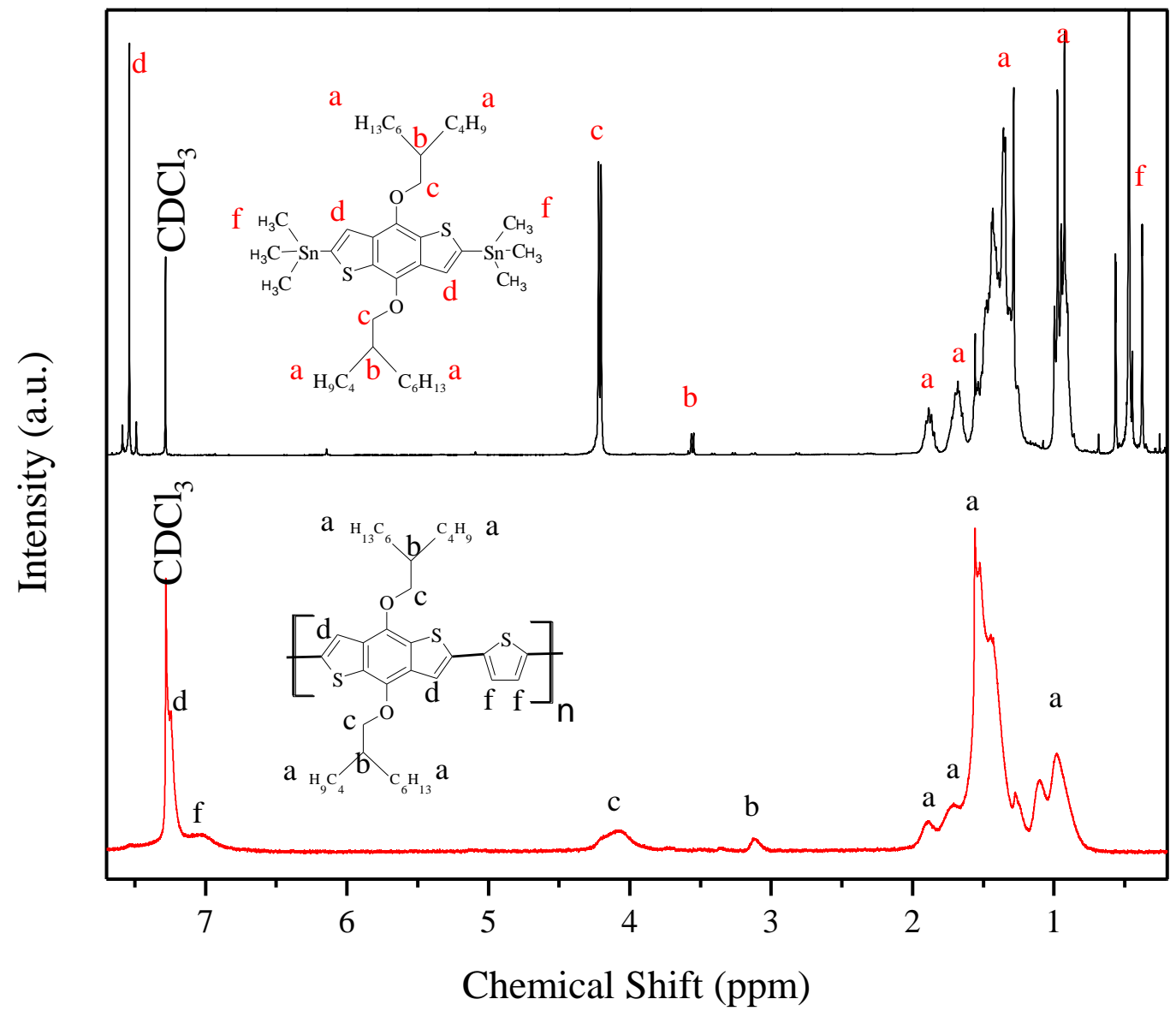

Figure 3. ${ }^{1} \mathrm{H}-\mathrm{NMR}$ spectra of BOBDT monomer (top) and its resulting PBOBDTTh polymer (bottom). 


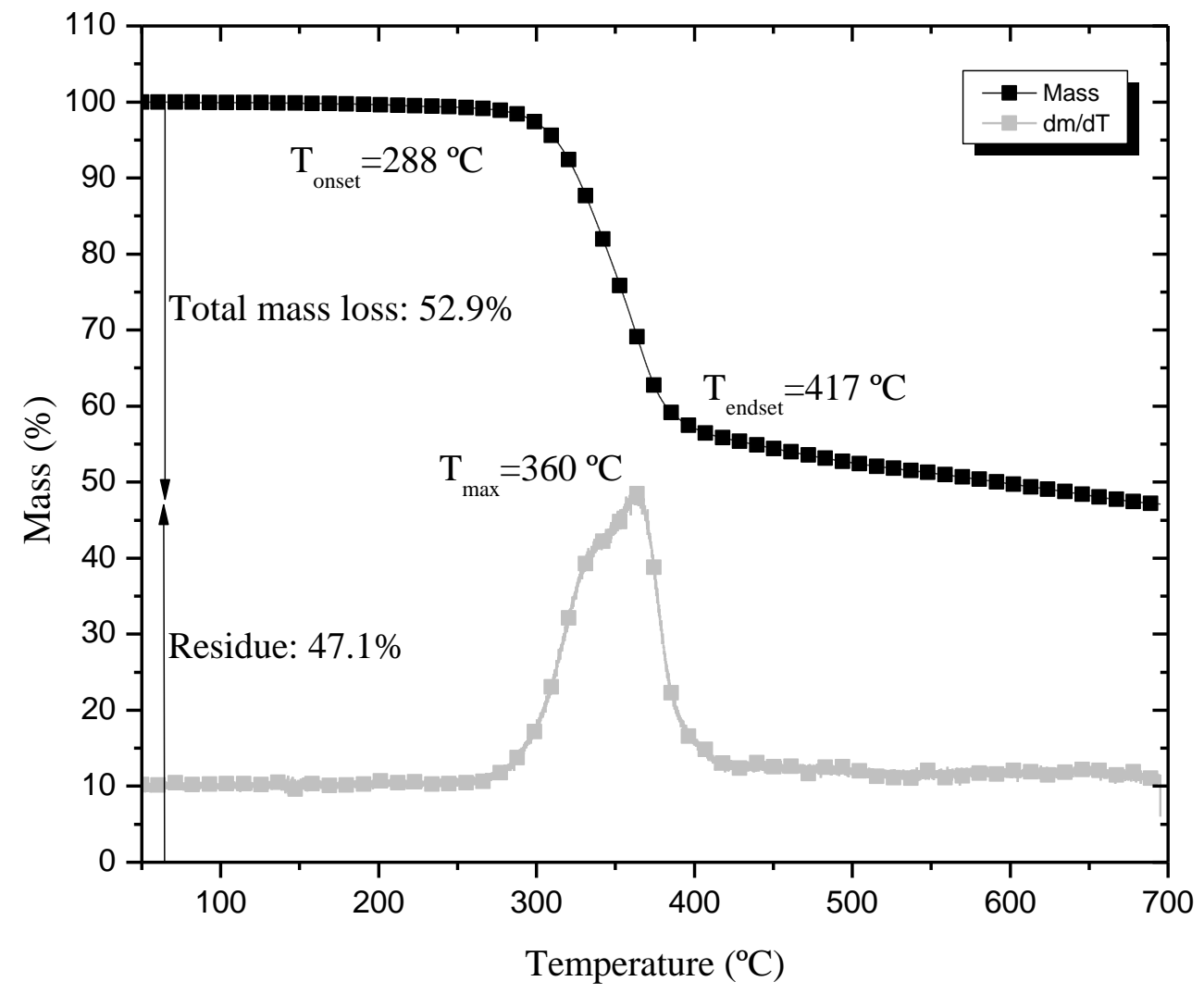

Figure 4. Thermogravimetric analysis for PBOBDTTh showing two different curves: the mass loss (top) and the differential curve (bottom). 


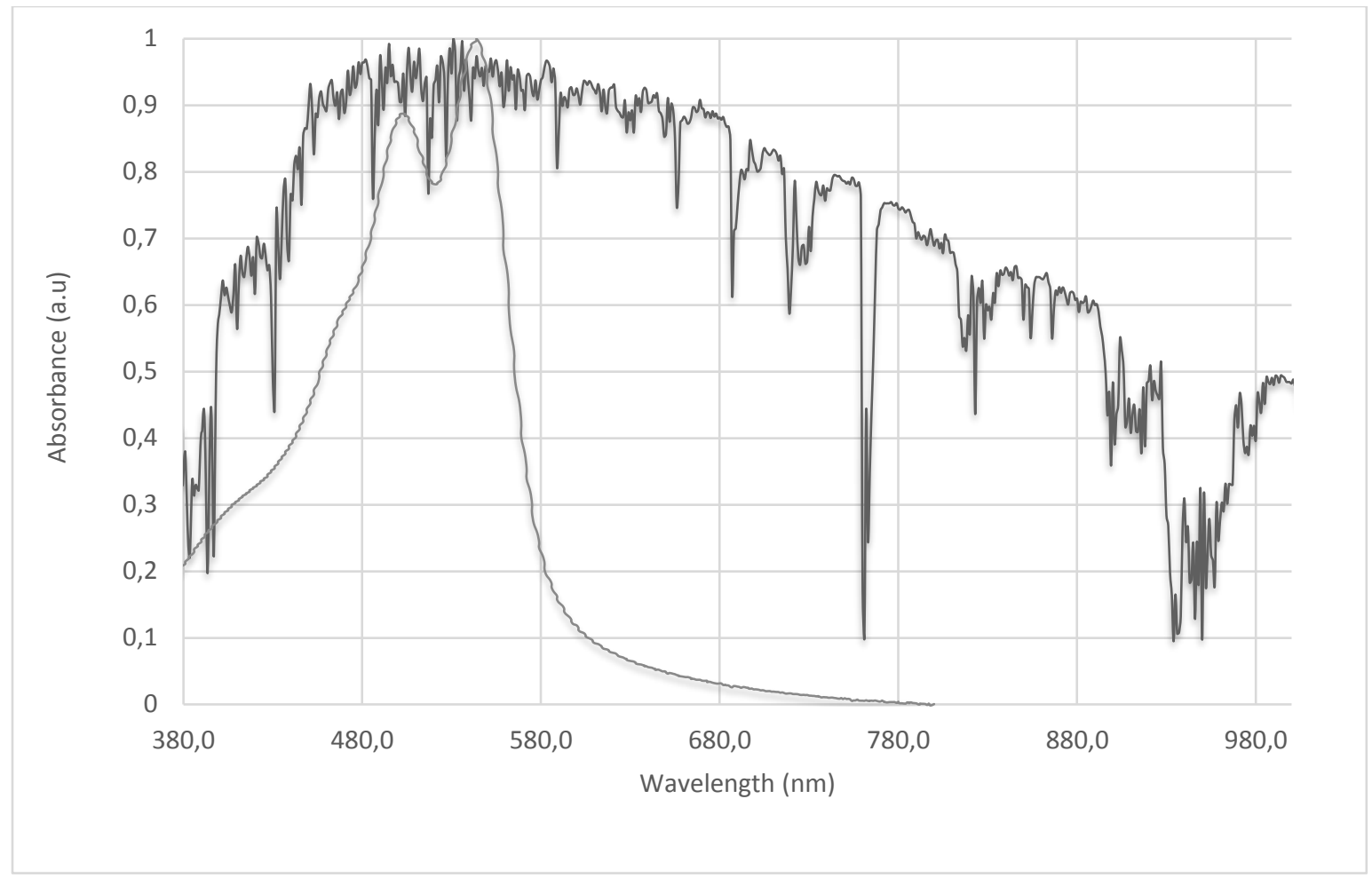

Figure 5. Absorption spectrum of the synthesized polymer (light) and the normalized solar irradiance spectrum AM1.5 (dark). 


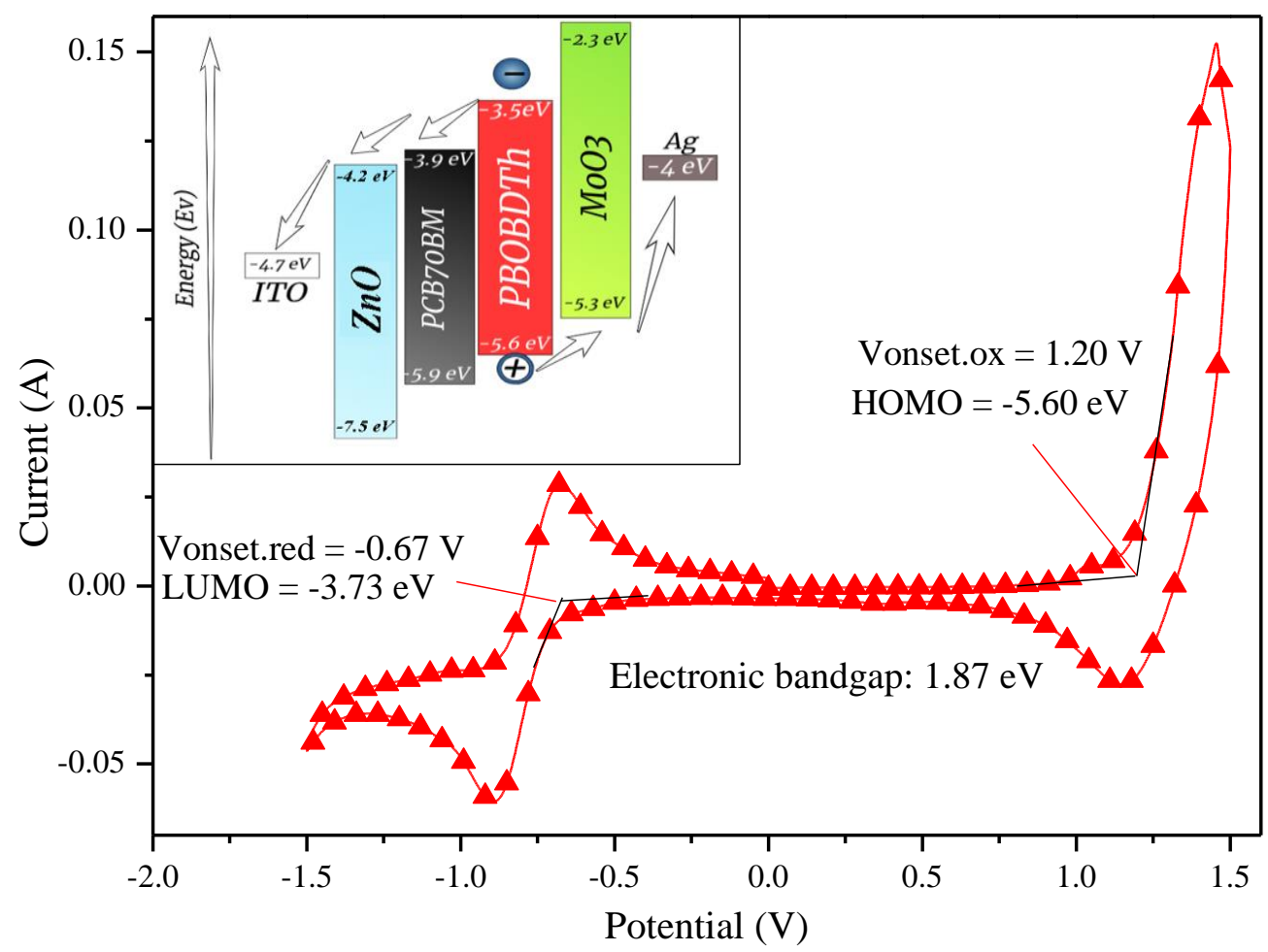

Figure 6. Cyclic voltammetry between -1.5 and $1.5 \mathrm{~V}$ for PBOBDTTh, graphic interpolation shows the estimation of reduction and oxidation onsets, equivalent to LUMO and HOMO energy levels, respectively. Inset image shows the resultant energy levels of an inverted device containing PBOBDTTh after applying the optical bandgap obtained through UV-vis and the HOMO level obtained through the CV. 


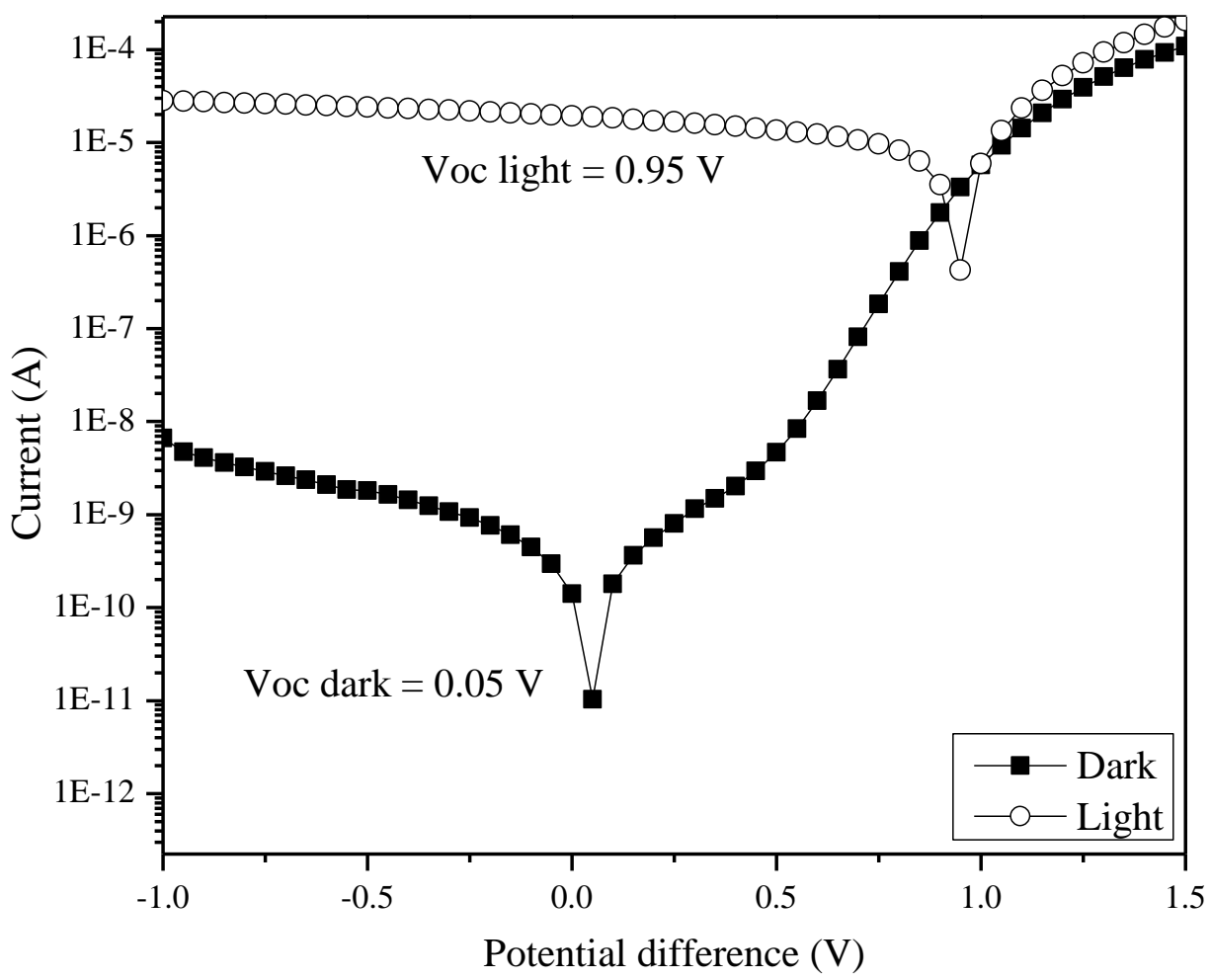

Figure 7. Current (log scale) as a function of potential difference for illuminated and non-illuminated ITO/ZnO/PC70BM:PBOBDTTh/ $\mathrm{MoO}_{3} / \mathrm{Ag}$ device. 


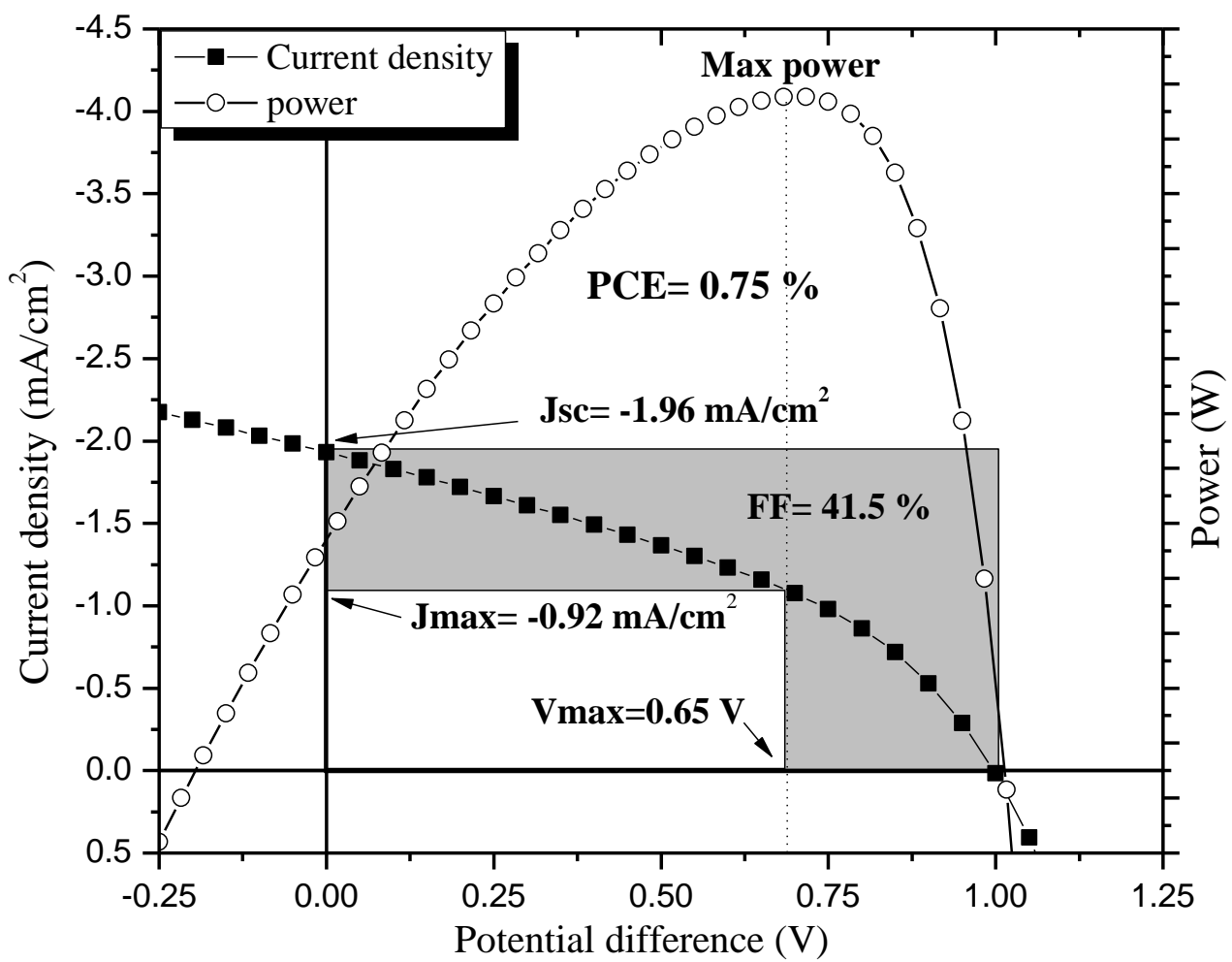

Figure 8. Photovoltaic parameters obtained through current density and power as a function of potential difference. 


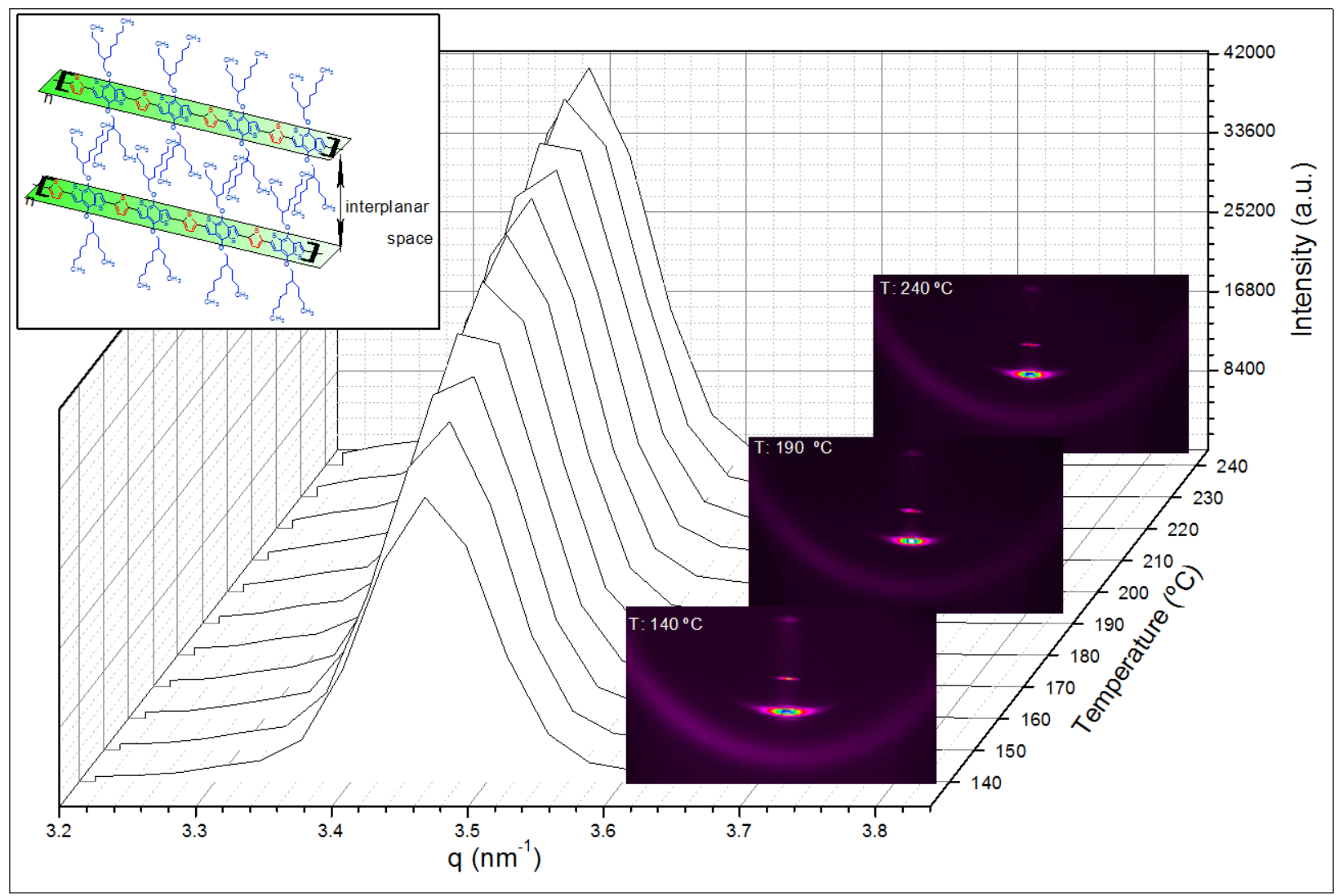

Figure 9. Evolution of the GIWAXS diffraction peak at low angles along with temperature for the thin film of pure PBOBDTTh. Inset image (top-left) shows the interplanar distance corresponding to the selected diffraction peak. The three inset images in the right-bottom corner are pictures of the scattering detected at the Pilatus $300 \mathrm{~K}$ detector. 


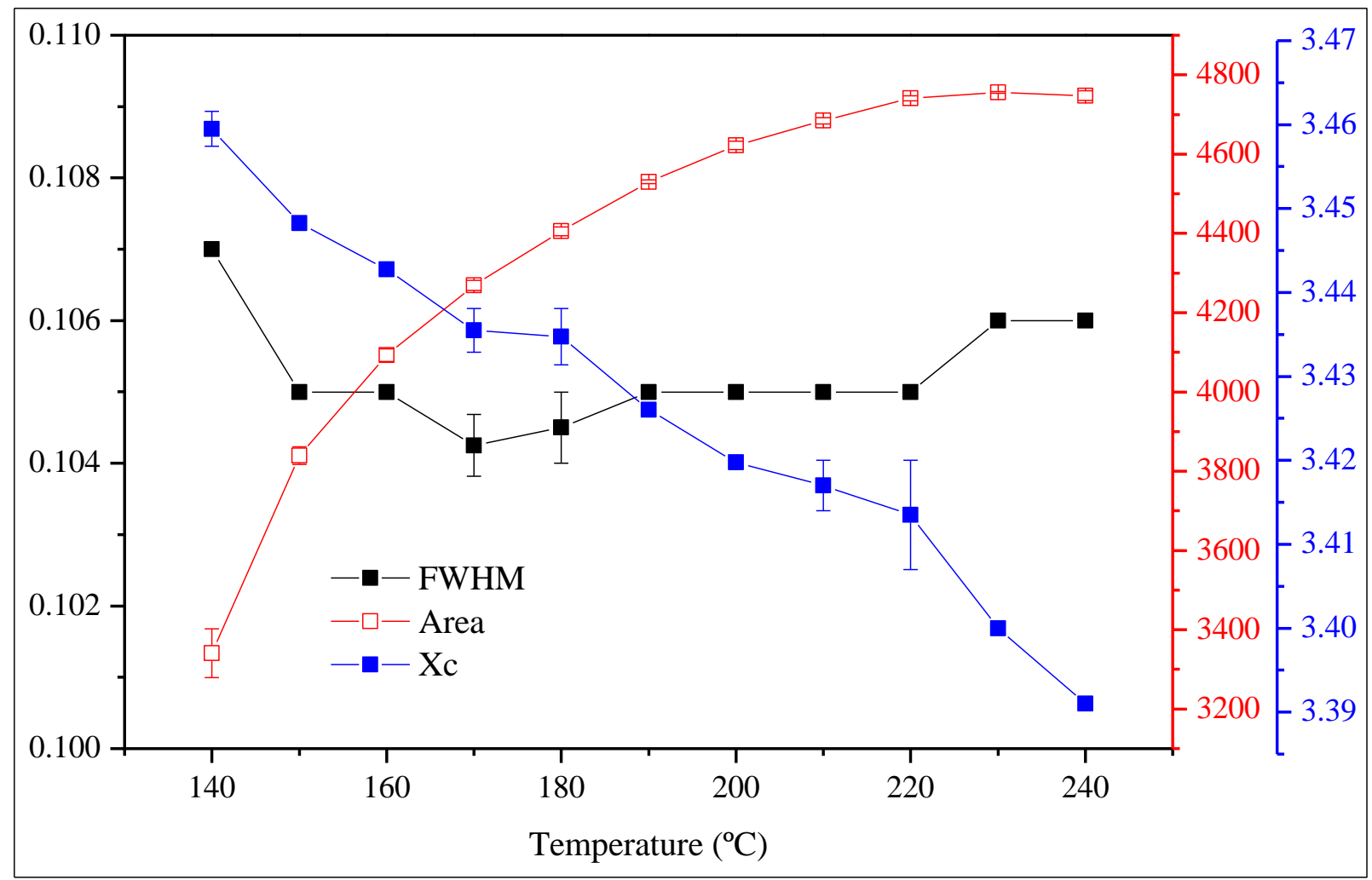

Figure 10. Evolution of FWHM, area of crystalline peak, and peak maximum $(\mathrm{Xc})$ as a function of temperature for a thin film of PBOBDTTh.

Table 1. Thermal and electrooptical properties of PBOBDTTh. Photovoltaic performance for a binary active layer with PC70BM 1:1 and 30mg/ml dichlorobenzene solution without additives and inverted architecture.

\begin{tabular}{|c|c|c|c|c|c|c|c|c|}
\hline $\begin{array}{c}\mathrm{M}_{\mathrm{n}}(\mathrm{kg} / \mathrm{mol}) \\
/ \mathrm{\oplus}\end{array}$ & $\begin{array}{c}\mathbf{T}_{\text {onset }} / \mathbf{T}_{\max } \\
\left({ }^{\circ} \mathbf{C}\right)\end{array}$ & $\begin{array}{c}\text { HOMO } \\
(\mathrm{eV})\end{array}$ & $\begin{array}{c}\text { LUMO } \\
(\mathrm{eV})\end{array}$ & $\begin{array}{c}\text { Bandgap } \\
(\mathrm{eV})\end{array}$ & $\begin{array}{c}\mathbf{J}_{\mathrm{sc}} \\
(\mathbf{m A} / \mathbf{c m})\end{array}$ & $\begin{array}{l}\mathbf{V}_{\text {oc }} \\
(\mathbf{V})\end{array}$ & $\begin{array}{l}\text { FF } \\
(\%)\end{array}$ & $\begin{array}{c}\text { PCE } \\
(\%)\end{array}$ \\
\hline $34.7 / 2.84$ & $288 / 360$ & -5.6 & -3.5 & 2.1 & -1.96 & 0.95 & 41.5 & 0.75 \\
\hline
\end{tabular}

